\title{
Serum Hepcidin Evaluation in Patients with Chronic Dialysis
}

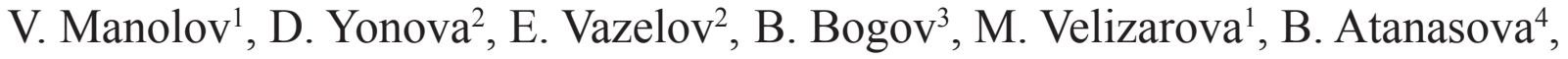 \\ V. Vasilev ${ }^{4}$, K. Tzatchev ${ }^{4}$, I. Bogov ${ }^{5}$ \\ ${ }^{1}$ Department of Medical Genetics, Medical University, Sofia, Bulgaria \\ ${ }^{2}$ Clinical Center of Dialysis, Medical University, Sofia, Bulgaria \\ ${ }^{3}$ Department of Clinical Nephrology, Medical University, Sofia, Bulgaria \\ ${ }^{4}$ Department of Clinical Laboratory and Clinical Immunology, Medical University, Sofia, Bulgaria \\ ${ }^{5}$ National Cardiological Hospital,Sofia, Bulgaria
}

\begin{abstract}
The aim of our study was to quantify serum hepcidin levels in Bulgarian patients with chronic kidney disease (CKD). Expected high values of peptide hormones might provide a new therapeutic choice for anemia of chronic disease. We looked for correlation between serum hepcidin levels and some iron metabolism parameters in CKD patients.

Results: The sandwich ELISA is highly specific for hepcidin-25. We found statistically significant differences in serum hepcidin levels in patients of the control group, with CKD stages II to IV and CKD stage V (on chronic dialysis): $12.7 \pm 8.7 \mu \mathrm{g} / \mathrm{L}$, $90.74 \pm 21.1 \mu \mathrm{g} / \mathrm{L}$, and $282.49 \pm 81.1 \mu \mathrm{g} / \mathrm{L}$, respectively. Significant correlation between serum hepcidin and transferrin saturation $(\mathrm{r}=0.340, P<0.05)$ was found in the group of patients with CKD stage $\mathrm{V}$.

Conclusion: The use of 2 monoclonal antibodies in a sandwich ELISA format provides a reliable, reproducible, and inexpensive method for measuring serum concentrations of the bioactive form of hepcidin in Bulgarian laboratory practice.

Key words: Hepcidin; anemia of chronic disease; chronic kidney disease (CKD); hemodialysis.

\section{Introduction}

Maintaining a balance of iron in the body is critical to the state of health. Identifying hepcidin as a key regulator of iron dramatically improves our understanding of the molecular control mechanisms of homeostasis and allows a more detailed understanding of the pathophysiology in clinical disorders. Recent studies highlight the role of hepcidin as a useful diagnostic tool and therapeutic target in various diseases with impaired iron exchange. Hepcidin is a 25 -aminoacid, cysteine-rich, iron-regulating peptide. Hepcidin quantification in human serum provides new topics for the pathogenesis of disorders of iron homeostasis and their treatment.

Hepcidin regulates systemic iron homeostasisabsorption in the duodenum, recycling demolition erythrocytes, controlled release from landfills in hepatocytes.

It is a peptide which is synthesized by the liver in response to

*Corresponding author: Victor Manolov, MD. Department of Medical Genetics, Medical University, Sofia, Bulgaria E-mail: victhedoc2@yahoo.com

a series of signals according to the needs of the body for iron. The biological action of hepcidin is mediated by its binding to the receptor ferroportin, which is the only known exporter of iron present in the duodenum, macrophages, hepatocytes, or placenta. Hepcidin binds to the receptor in general complex, is internalized, and unlocks the lysosomal degradation ferroportin structure [1-4].

Anemia of chronic disease (ACD), also known as anemia of inflammation, is the most common type of anemia in hospitalized patients worldwide. It is observed in patients with acute or chronic inflammatory diseases, infections, cancer, rheumatoid arthritis, chronic kidney disease (CKD), organ failure, and trauma [1]. Anemia is usually mild or moderate, often without clear changes in the morphological characteristics of red blood cells. The pathogenesis is associated with induced hepcidin synthesis due to the catalytic effect of the inflammatory cytokines, of which the most important is interleukin-6 (IL-6). High hepcidin levels are cause for reduced intestinal absorption of iron and difficult release of the macrophages, leading to subsequent hypoferremia and suppressed erythropoesis.
\end{abstract}


The aim of our study was to quantify serum hepcidin levels in Bulgarian patients with CKD. Expected high values of peptide hormones might provide a new therapeutic choice for anemia of chronic disease. We looked for correlation between serum hepcidin levels and some iron metabolism parameters in CKD patients.

\section{Material and Methods}

This study included 55 healthy volunteers and 60

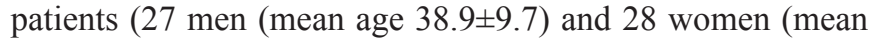
age 41.3 \pm 8.3 )) with CKD stages II to $\mathrm{V}$, including $32 \mathrm{CKD}$ patients on chronic dialysis. Staging of the disease was performed using eGFR as recommended by the CKD-EPI Creatinine Equation. All enrolled subjects filled out informed consent according to the Helsinki Declaration (Directive 2001/20/EC).

The study analyzed serum from individuals included in the study. All samples were stored at $-70^{\circ} \mathrm{C}$ until determination of serum hepcidin. Characterization of iron metabolism parameters was determined using an automatic biochemical analyzer Cobas Integra 400 (of Roche Diagnostics) and Advia 2120 hematology analyzer (Siemens Healthcare Diagnostics). We used the verified ELISA method to quantify serum hepcidin levels [5,6]. Data were analyzed by the Student t-test. Pearson's Correlation Coefficient (r) was used to determine the strength of the relationship between the two continuous variables. A probability value of $P<0.05$ was considered statistically significant.

\section{Results and Discussion}

We found statistically significant differences in serum hepcidin levels in patients with CKD stages II to IV and CKD stage $\mathrm{V}$ (on chronic dialysis) versus the control group: $90.74 \pm 21.1 \mu \mathrm{g} / \mathrm{L}, \quad 282.49 \pm 81.1 \mu \mathrm{g} / \mathrm{L}, \quad$ and $\quad 12.7 \pm 8.7 \mu \mathrm{g} / \mathrm{L}$, respectively $(P<0.01)$. We looked for a correlation between the analyzed parameters for the iron deficiency in CKD and hepcidin (Table 1). Significant correlation between serum hepcidin and transferrin saturation $(\mathrm{r}=0.340, P<0.05)$ was found in the group of patients with CKD stage $\mathrm{V}$.

Table 1.

Correlation between hepcidin and the measured parameters

\begin{tabular}{|l|c|c|c|c|}
\hline \multirow{3}{*}{ Parameter } & \multicolumn{5}{c|}{ Hepcidin } \\
\cline { 2 - 5 } & CKD (stages II to IV) & \multicolumn{2}{c|}{ CKD (stage V) } \\
\cline { 2 - 5 } & $\mathrm{r}$ & $P$ & $\mathrm{r}$ & $P$ \\
\hline PCR & 0.155 & $<0.05$ & n.a. & n.a. \\
\hline eGFR & -0.297 & $<0.05$ & -0.083 & $<0.05$ \\
\hline MCV $(\mathrm{fl})$ & 0.139 & $>0.5$ & 0.372 & $<0.05$ \\
\hline Iron $(\mu \mathrm{mol} / 1)$ & 0.088 & $<0.05$ & 0.204 & $<0.05$ \\
\hline TSAT $(\%)$ & 0.156 & $<0.05$ & 0.340 & $<0.05$ \\
\hline TIBC $(\mu \mathrm{mol} / 1)$ & -0.089 & $<0.05$ & -0.235 & $<0.05$ \\
\hline Reticulocytes $(\%)$ & 0.012 & $<0.05$ & -0.268 & $<0.05$ \\
\hline
\end{tabular}

$P C R$ - protein to creatinine ratio; $M C V$ - middle cell volume;

TSAT - transferrin saturation; TIBC - total iron binding capacity.
Development of erythropoesis stimulating agents (ESA) such as erithropetin allows effective therapy in patients with CKD. The optimal target hemoglobin concentration is subjected to various discussions; during the treatment a significant number of patients become resistant to erythropoesis stimulating agents. Soon after its discovery, hepcidin was closely associated with CKD because of its role in advancing homeostasis imbalance between iron and resistance to ESA $[7,8]$. As such, hepcidin was described as a marker that could be used to predict response to the ESA, and lead clinicians in conducting therapy with the ESA and intravenous iron. Later this role was well recognized in practice.

$\mathrm{ACD}$ is an acquired disorder of iron homeostasis. This condition is associated with infection, organ failure, severe trauma, or other causes of inflammation. Anemia is usually mild or moderate; the erythrocytes cannot show typical characteristics of iron deficiency. This type of anemia is caused by induced hepcidin synthesis due to the action of interleukin-6 and other cytokines. According to some authors, normocytic erythrocytes in anemia of chronic diseases are the result of the action of two opposite effects: on the one hand, insufficient iron, and on the other, a tendency to macrocytosis that is still unknown in detail but is probably due to abnormal folate homeostasis in response to inflammation [9]. There are currently no laboratory tests that can fully distinguish ACD of iron deficiency anemia (IDA) [10,11]. Differentiation between those two states is often achieved by a combination study of various biochemical markers of iron metabolism. ACD is a heterogeneous disease that is typically characterized by normocytic anemia, alterations in the erythropoietic response, low serum iron, lower transferrin saturation, and iron accumulation in macrophages [1]. It is important to note that the functional iron deficiency in $\mathrm{ACD}$ is different from the actual iron deficiency in IDA, where the iron is exhausted due to the circulation and macrophages [12]. Some studies over the past 10 years have shown that functional iron deficiency in ACD may be due to increased levels of the regulatory hormone hepcidin.

Pre-clinical and clinical studies have shown that hepcidin antagonists, such as antibodies against hepcidin, antibodies to cytokine receptors, and the stabilizing hypoxia inducible factor (HIF), can lead to a reduction in hepcidin over-expression and can correct the pathological changes in the iron status [13-15]. BMP-2 administration increases hepcidin expression and decreases serum iron levels in vivo [13]. In $\mathrm{Hfe}^{-/}$mice, supraphysiologic doses of exogenous BMP6 improved hepcidin deficiency, reduced serum iron, and redistributed tissue iron to appropriate storage sites [16]. In order to suppress tumor angiogenesis HIF antagonists are used in malignancies [17]. Blocking of HIF could increase the concentration of hepcidin in conditions of iron overload.

New knowledge on the role of hepcidin in the development of anemia in CKD can significantly contribute to the correct choice of therapeutic approach [18].

\section{Conclusion}

Implementation in clinical laboratory practice as a routine method for the study of plasma concentrations of 
hepcidin is a step forward in the treatment of impaired iron homeostasis. It would allow improving the differentiation between iron-deficiency anemia and anemia of chronic disease. The use of 2 monoclonal antibodies in a sandwich ELISA format provides a reliable, reproducible, and inexpensive method for measuring serum concentrations of the bioactive form of hepcidin in Bulgarian laboratory practice.

\section{Competing interests}

The authors declare that they have no competing interests.

\section{Acknowledgements}

This study was conducted with the support of the Medical University of Sofia - Grant № 10/2013.

\section{References}

1. Sun CC, Vaja V, Babitt JL, Lin HY. Targeting the hepcidinferroportin axis to develop new treatment strategies for anemia of chronic disease and anemia of inflammation. Am J Hematol 2012; 87(4):392-400.

2. Ganz T, Nemeth E. Iron sequestration and anemia of inflammation. Semin Hematol 2009; 46(4):387-393.

3. Ganz T. Hepcidin and iron regulation, 10 years later. Blood 2011; 117(17):4425-33.

4. Ramey G, Deschemin JC, Durel B, Canonne-Hergaux F, Nicolas G, Vaulont S.. Hepcidin targets ferroportin for degradation in hepatocytes. Haematologica 2010; 95(3):501-4. 5. Manolov V, Atanasova B, Velizarova M et al. Hepcidin evaluation in biological fluids. Medical Review 2013; XLIX(2): 41-46.

6. Manolov V, Atanasova B, Velizarova M, Vasilev V, Tzatchev K. Serum hepcidin levels in Bulgarian population. Clin Lab 2014; 60(12):2001-6.

7. Nicolas G, Viatte L, Bennoun M, Beaumont C, Kahn A,
Vaulont S. Hepcidin, a new iron regulatory peptide. Blood Cells Mol Dis 2002;29(3):327-35.

8. Swinkels DW, Wetzels JF. Hepcidin: a new tool in the management of anaemia in patients with chronic kidney disease? Nephrol Dial Transplant 2008;23(8):2450-3.

9. Andrews NC. Forging a field: the golden age off iron biology. Blood 2008; 112(2):219-30

10. Hentze MW, Muckenthaler MU, Galy B, Camaschella C. Two to tango: regulation of Mammalian iron metabolism. Cell 2010; 142(1):24-38.

11. Kroot JJ, Tjalsma H, Fleming RE, Swinkels DW. Hepcidin in human iron disorders: diagnostic implications. Clin Chem 2011; 57(12):1650-69.

12. Theurl I, Aigner E, Theurl M, Nairz M, Seifert M, Schroll A, et al. Regulation of iron homeostasis in anemia of chronic disease and iron deficiency anemia: diagnostic and therapeutic implications. Blood 2009;113(21):5277-86.

13. Babitt JL, Huang FW, Xia Y, Sidis Y, Andrews NC, Lin HY.. Modulation of bone morphogenetic protein signaling in vivo regulates systemic iron balance. J Clin Invest 2007;117(7):1933-9.

14. Sasu BJ1, Cooke KS, Arvedson TL, Plewa C, Ellison AR, Sheng J, et al. Antihepcidin antibody treatment modulates iron metabolism and is effective in a mouse model of inflammationinduced anemia. Blood 2010;115(17):3616-24.

15. Fatih N, Camberlein E, Island ML, Corlu A, Abgueguen E, Détivaud L, et al. Natural and synthetic STAT3 inhibitors reduce hepcidin expression in differentiated mouse hepatocytes expressing the active phosphorylated STAT3 form. J Mol Med (Berl) 2010; 88(5):477-86.

16. Corradini E, Schmidt PJ, Meynard D, Garuti C, Montosi $\mathrm{G}$, Chen S, et al. BMP6 treatment compensates for the molecular defect and ameliorates hemochromatosis in Hfe knockout mice. Gastroenterology 2010;139(5):1721-9.

17. Semenza GL. Development of novel therapeutic strategies that target HIF-1. Expert Opin Ther Targets 2006;10(2):267-80. 18. Yonova D, Vazelov E, Manolov V, et al. Diagnostic tools of hepcidin in dialysis patients. Neph Dial Transpl (Bulg) 2013; 19(3): 22-7. 